Check for updates

Cite this: J. Mater. Chem. A, 2021, 9, 27361

Received 14th September 2021 Accepted 2nd November 2021

DOI: $10.1039 / d 1 t a 07897 f$

rsc.li/materials-a

\section{Mechanochemical synthesis of sodium carboxylates as anode materials in sodium ion batteries $\dagger$}

\author{
Daniel N. Rainer, (D) a Aamod V. Desai, (D) ab A. Robert Armstrong (D) *ab \\ and Russell E. Morris (D)*ab
}

\begin{abstract}
The ever-growing global energy demand necessitates, amongst other technologies, advances in materials for electrochemical energy storage such as sodium ion batteries. The recent advent of organic-based electrodes is driven by their tremendous structural versatility and the great potential in developing a green battery cycle. Current research aims to solve remaining obstacles such as a lack of robust, efficient and scalable synthesis procedures. In this vein, we present a fast and sustainable mechanochemical synthesis route towards four sodium carboxylates compounds. Target materials can be obtained in the substantially decreased reaction time of only one hour, while retaining the good electrochemical performance reported for conventionally synthesised compounds. More importantly, no solvent is required for these mechanosynthetic transformations, making this approach attractive with respect to goals in line with green chemistry as well as from an economical point of view. The variety of synthesised compounds hints at possible generalisability of the developed methodology and its potential applicability for many other known compounds, beyond the sphere of rechargeable battery systems.
\end{abstract}

\section{Introduction}

Global energy demand has been increasing steadily in recent years and technological advances are needed to help meet these demands. ${ }^{1}$ One aspect of this goal is to find solutions for energy storage and their subsequent deployment including rechargeable batteries. The currently most commonly used class of these are lithium ion batteries (LIB) which find use for example in portable devices (mobile phones, laptops, ...) and electric vehicles. ${ }^{2}$ In an effort to find alternatives to lithium, research has extended to sodium and potassium ion batteries (NIB, KIB) which are based on much more abundant elements. Despite the higher molecular mass of sodium containing battery materials compared to their counterparts in LIBs, they hold promise with respect to lower production costs and increased availability, while retaining the advantages of alkali metal ion batteries (AMIB). ${ }^{3-6}$

Traditionally, the materials that are utilised in electrodes of AMIBs are metal oxides or carbons such as graphite..$^{7-10}$ Although these electrodes perform very well, they come at a price. Oxide-based positive electrodes for LIBs typically

${ }^{a}$ EastCHEM School of Chemistry, University of St Andrews, North Haugh, KY16 9ST, St Andrews, UK.E-mail: ara@st-andrews.ac.uk; rem1@st-andrews.ac.uk

${ }^{b}$ The Faraday Institution, Quad One, Harwell Science and Innovation Campus, Didcot, OX11 ORA, UK

$\dagger$ Electronic supplementary information (ESI) available. See DOI: 10.1039/d1ta07897f contain cobalt, which is accompanied by severe environmental concerns. For the negative electrode, carbon-based compounds are by far the most used active component. In LIBs, graphite shows strong performance with insertion of lithium ions between the graphene layers. In the case of sodium, however, graphite is unfeasible, due to the size mismatch of the ions and the interlayer spacing. ${ }^{11}$ This obstacle is the main driving force for the quest to find a suitable anode material in NIBs. ${ }^{12,13}$ Popular alternatives are related carbon-based materials generally referred to as 'hard carbon'. ${ }^{\mathbf{1 4 1 5}}$ These amorphous, mechanically durable solids feature several advantageous properties both in terms of availability and electrochemical performance. Although these materials show great promise, drawbacks in their production, involving high temperatures, as well as issues regarding long term cyclability of batteries based on hard carbon warrant further development of suitable materials. Additionally, the high porosity and surface area of hard carbon necessitate larger volumes of electrolyte for sufficient coverage. This comes with disadvantages in terms of cost but more importantly a significant drop in performance due to a considerable irreversible contribution to the discharge capacity for initial cycles. Overcoming these issues, the field has seen increased interest in the possibility of using organic based electrodes. ${ }^{16-22}$ Several key advantages can be discerned: (1) immense structural flexibility through the wide range of available and synthesizable chemicals; (2) sustainable production of such reagents from biomass; (3) lower environmental impact 
compared to (transition) metal oxide-based materials with potentially hazardous components.

For a fully functioning anode, the organic material needs to be complemented with an electrically conductive material, in most cases simply conductive carbon. Homogeneous distribution is achieved by combining them using a binder. There are many available choices; however, water-based binders like sodium carboxymethylcellulose (CMC) or sodium alginate are favourable when considering the health and safety concerns regarding traditionally used components like the mutagenic PVDF (polyvinylidene difluoride) and the concomitantly used solvents such as NMP ( $N$-methyl pyrrolidone).$^{23}$ Fortunately, most organic-based anodes are compatible with environmentally benign water-soluble binder alternatives, once more increasing their value as candidate materials for industrialscale production and widespread use.

The demand for environmentally friendly methods in chemistry has seen the re-discovery of mechanical methods as a driving force in chemical synthesis. ${ }^{24-26}$ Ball milling is probably the most used technique in laboratories, combining a simple setup with the potential for facile upscaling. Compared to manual grinding it has the advantage of exerting much higher forces and high reproducibility. The reagents are milled together using a milling vessel and media made from appropriate materials so as not to interfere with the reaction itself. The high impact of the balls on the reagents facilitates chemical bond breaking and making, leading to new compounds. The reactions are typically much faster compared to an analogous solution-based synthesis. Additionally, the transformation is carried out solvent-free, which means not only savings in terms of cost, but more importantly a significant reduction of produced waste. Mechanochemical synthesis has now been reported in many areas including organic synthesis, ${ }^{27,28}$ inorganic and main group chemistry, ${ }^{29,30}$ materials science, ${ }^{31-33}$ and medicinal chemistry. ${ }^{34}$ There have also been several reports of employing mechanical forces for the production of inorganic anode materials for batteries..$^{35-37}$ In contrast, organic compounds for use in energy storage applications have not been investigated so far. The mechanochemical synthesis of coordination compounds based on carboxylic acids has been studied mostly in the construction of metal-organic frameworks (MOFs) where these organic molecules function as linkers between metal(-cluster) nodes. ${ }^{38-40}$ Traditionally, MOFs are produced through crystallization out of a solution containing both metal ions and the dissolved organic compound. The possibility of making these highly porous compounds with applications in gas storage, drug delivery, and catalysis through mechanochemical means renders them highly attractive from an industrial and large-scale production point of view. ${ }^{\mathbf{4 1}}$

Coordination polymers of alkali metal ions and carboxylates (lithium, sodium, and potassium salts of carboxylic acids) are an attractive class of materials for anodes in AMIBs. ${ }^{16,42,43}$ The carboxylate functionality is redox active as it can undergo a reduction, enabling insertion of additional alkali-metal ions. Stabilization of this negatively charged state is facilitated by the presence of a conjugated $\pi$-system in the organic backbone such as aromatic rings or conjugated alkenes. Several materials of this type such as sodium terephthalate ${ }^{\mathbf{4 4 - 4 6}}$ have been reported to be suitable active components in negative electrodes. ${ }^{\mathbf{1 6 , 1 8 , 4 7}}$ The preparation of these compounds is typically conducted using corrosive sodium hydroxide as well as large amounts of solvent. Additionally, they usually require up to $24 \mathrm{~h}$ of reaction time as well as heating, sometimes under reflux conditions. These circumstances have been a hindrance regarding large-scale processing of such materials in an industrial setting. Furthermore, there are also several concerns with respect to environmental impact and energy consumption. Fortunately, all these issues can be addressed by employing mechanochemical methods for the synthesis of the target compounds.

Sodium dicarboxylates have been reported as promising active compounds for anodes of sodium ion batteries (NIBs). ${ }^{\mathbf{1 6}}$ Taking inspiration from compounds that have been synthesised using solution-based methods, several dicarboxylic acids were selected as potential candidate reagents (Fig. 1). ${ }^{\mathbf{4 4 6 , 4 8 - 5 0}}$ Besides the carboxylic acid functionality, these compounds have interesting chemical differences in their backbone that should result in different electrochemical behaviour. In contrast to conventional syntheses, mechanochemical methods feature several advantages, such as low amounts or avoidance of solvents, decreased reaction time, waste reduction and atom efficient protocols.

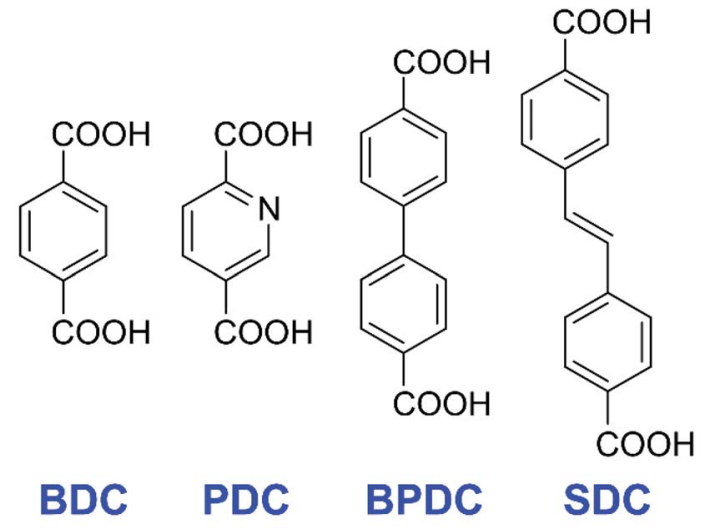

Synthesis

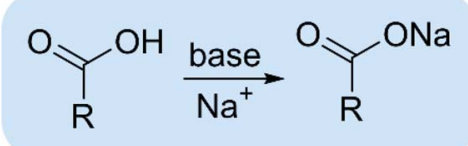

Electrochemistry

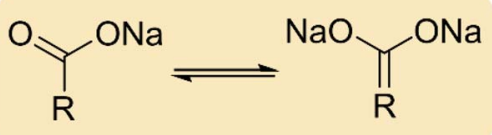

Fig. 1 Selected dicarboxylic acids to be used in the mechanochemical reaction to form sodium carboxylates for anodes in sodium ion batteries. BDC: 1,4-benzenedicarboxylic acid; PDC: 2,5-pyridinedicarboxylic acid; BPDC: 4,4'-biphenyldicarboxylic acid; SDC: 4,4'-stilbenedicarboxylic acid. 
The reaction of the carboxylic acids towards their sodium salt derivatives hinges on the deprotonation of the carboxylate group, which can then coordinate to the sodium cation. In conventional protocols, this is facilitated by employing sodium hydroxide, as it not only functions as the source of the alkali metal but also as a $\mathrm{pH}$ mediating agent. The resulting solution is highly basic; ideal conditions to abstract the proton from acid groups. These highly alkaline conditions coupled with the corrosive reagent itself, however, bring with them concerns in terms of practical handling and safety. In our mechanochemical ball milling approach, we employ benign sodium acetate as the reagent, both in its hydrated as well as anhydrous form. The acetate salt used here is slightly basic, which, combined with the exerted forces from the milling balls, is shown to be sufficient for the transformation of the carboxylic acids to the respective carboxylates.

We present a fast and efficient method for the synthesis of sodium dicarboxylate compounds using mechanochemistry and their performance as electrodes in sodium ion batteries (NIB) is demonstrated (Fig. 2). Non-hazardous sodium acetate and a range of organic dicarboxylic acids such as 1,4-benzene dicarboxylic acid (BDC, terephthalic acid) were milled together using a shaker ball mill, resulting in crystalline coordination compounds. Such a mechanochemical reaction setup ensures facile preparative work and enables rapid conversion of the reagents, in some cases even within minutes. Besides the improvements in required time to prepare the target compounds, using this methodology is also beneficial with respect to amounts of liquid used, as there is no solvent necessary, leading to drastically reduced amounts of waste. Products were utilised as active materials for anodes in sodium ion batteries with performances equal to those of conventionally synthesised compounds. The variety of carboxylic acids used demonstrates the robustness of the method and can be considered precedential for production of functional organic materials for batteries.

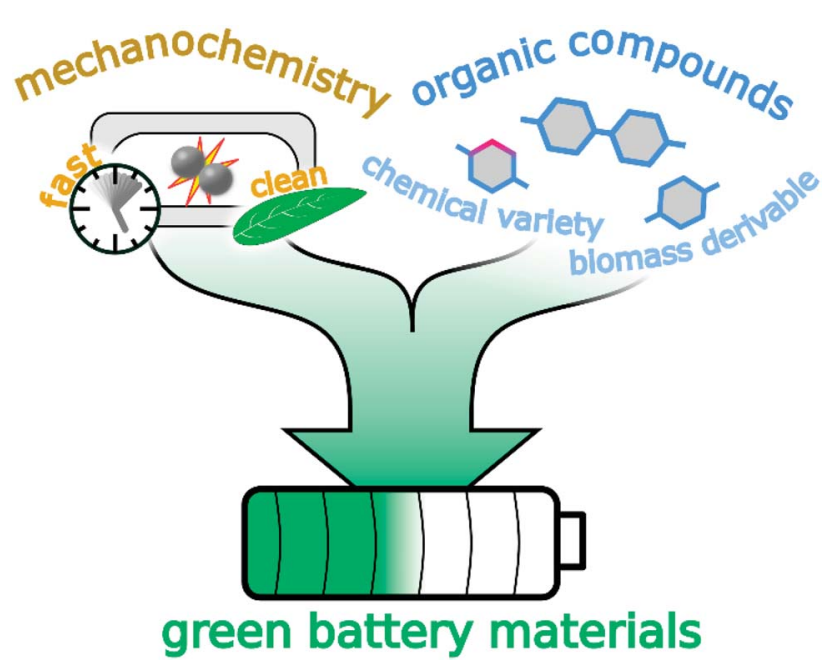

Fig. 2 Schematic representation of the combination of mechanochemical synthesis and organic compounds for the preparation of sustainable anode materials in sodium ion batteries.

\section{Experimental}

\section{Mechanochemical synthesis of sodium carboxylates}

The synthesis of sodium carboxylate compounds was conducted in a Retsch MM 400 Shaker mill operated at $30 \mathrm{~Hz}$ and using home-made polycarbonate milling jars (based on technical drawings in ref. 51) with stainless steel milling balls. Standard experiments were conducted using jars with an internal volume of $14 \mathrm{~mL}$ and two balls of $7 \mathrm{~mm}$ diameter $(1.3 \mathrm{~g})$ each and the milling experiment conducted for $60 \mathrm{~min}$. Larger quantities were produced using $25 \mathrm{~mL}$ jars and two balls of $12 \mathrm{~mm}$ diameter $(7 \mathrm{~g})$ each. Reagents in the desired ratio were weighed and placed into one jar half, together with the milling media. If additional liquid was required, it was added to the second half of the jar just before the start of the experiment. Obtained materials are scraped out of the jar using plastic spatulas and dried in an oven at $80^{\circ} \mathrm{C}$ overnight. An additional washing step was conducted for selected samples using ethanol. The washed sample was recovered by centrifugation and subsequently dried as above. Details for each experiment can be found in the ESI Table S1.†

\section{Elemental analysis}

Calculated for Na-BDC (monosodium): C, 51.1; H, 2.7. Found: C, 50.9; $\mathrm{H}, 2.6$.

Calcd for Na-BPDC (disodium): C, 58.8; H, 2.8. Found: C, 54.8; $\mathrm{H}, 3.2$.

Calcd for Na-SDC (disodium): C, 61.6; H, 3.2. Found: C, 62.6; $\mathrm{H}, 3.5$.

Calcd for Na-PDC (disodium): C, 39.8; H, 1.4; N, 6.6. Found: C, 39.6; H, 1.6; N, 6.1.

\section{Powder X-ray diffraction (PXRD)}

PXRD analysis was conducted on a PANalytical Empyrean diffractometer in Bragg-Brentano geometry in $\theta-2 \theta$ mode or on a STOE STADI-P diffractometer in Debye-Scherrer geometry. Both diffractometers employ $\mathrm{Cu} \mathrm{K} \alpha 1$ radiation (primary beam monochromator), and patterns were collected from $3-50^{\circ} 2 \theta$.

\section{Scanning electron microscopy (SEM)}

SEM was performed using a JEOL JSM-IT200 or a JEOL JSMIT800 microscope. Prior to analysis, samples were placed on a conductive carbon tape and coated with gold using a Quorum Q150R ES sputter coater (10 mA, $30 \mathrm{~s})$.

\section{Fourier-transform infrared spectroscopy (FTIR)}

FTIR spectra were obtained using a Shimadzu Affinity-1 and recorded in a range of $500-4000 \mathrm{~cm}^{-1}$.

\section{Thermogravimetric analysis (TGA)}

TGA was performed on a Stanton Redcroft STA-780 in air (25 $\mathrm{mL} \mathrm{min}^{-1}$ ), heating the sample up to $700^{\circ} \mathrm{C}$ with a ramping rate of $5{ }^{\circ} \mathrm{C} \min ^{-1}$. 


\section{Electrochemical characterization}

The working electrodes for Na-BDC, Na-PDC, Na-SDC, and NaBPDC were prepared by mixing active material (60\%), conductive carbon (Super C65, 30\%) and carboxymethyl cellulose (CMC, 10\%) as the binder. The slurry was prepared by hand grinding active material and conductive carbon, which was added to a CMC solution in water and stirred for $4 \mathrm{~h}$. The slurry was cast onto aluminium foil (Advent Research) using a doctor blade and air dried for $2 \mathrm{~h}$. Electrodes were then punched (diameter $\sim 12 \mathrm{~mm}$ ) and dried overnight in a vacuum oven at $110{ }^{\circ} \mathrm{C}$. The approximate active mass loading per electrode was $1.81,1.62,1.63$ and $1.66 \mathrm{mg} \mathrm{cm}^{-2}$ for Na-BDC, Na-PDC, Na-SDC, and Na-BPDC, respectively. Solid state characterisation for the air-dried electrode phase was performed by scraping off the solid mixture from the Al-foil. Coin cells (CR2325) were assembled using sodium metal (Sigma-Aldrich) as the counter electrode, glass fibre separator (Whatman GF/B) and $\mathrm{NaPF}_{6}$ in ethylene carbonate (EC) and diethyl carbonate (DEC) (1:1) (Kishida) as the electrolyte, in an argon-filled glovebox (MBraun) with oxygen and water content $<1$ ppm. Electrochemical studies were performed at $30{ }^{\circ} \mathrm{C}$ in a potential window of 2.5-0.01 V ( vs. Na/ $\mathrm{Na}^{+}$) on a Biologic BCS-805 modular battery testing system and the data were analysed using BT-Lab software.

\section{Results and discussion}

\section{Mechanochemical synthesis of sodium benzenedicarboxylate}

Initial experiments were conducted using 1,4-benzenedicarboxylic acid (BDC), which has been reported to yield materials exhibiting excellent electrochemical performance. ${ }^{\mathbf{4 4 - 4 6}}$ Sodium acetate trihydrate and BDC were milled together in various metal/linker ratios $(\mathrm{M} / \mathrm{L})$ employing a commercial shaker ball mill operated at $30 \mathrm{~Hz}$. A Na-BDC phase was obtained in good purity when using $\mathrm{M} / \mathrm{L}=1$, whereas higher ratios only led to a greater proportion of unreacted starting material (compare Fig. S1†). This phase corresponds to a monosodium terephthalate $^{\mathbf{4 6 , 5 2}}$ where the second carboxylic acid group has not been deprotonated (Fig. 3a). A small amount of residual sodium acetate can be observed in the PXRD pattern and may be removed by washing with small portions of ethanol. This purification step, however, is not necessary as shown below in electrochemical tests, where the material obtained from the milling operation was used directly in the preparation of electrodes.

PXRD analysis also indicates unreacted terephthalic acid (peak at circa $17.4^{\circ}$ ), however FTIR spectroscopy does not show any bands at high wavenumbers $\left(>3000 \mathrm{~cm}^{-1}\right)$, which would correspond to $\mathrm{O}-\mathrm{H}$ stretch vibrations from free carboxylic acid groups (Fig. 3c). This indicates that no uncoordinated carboxylic acid remains. A band at $1700 \mathrm{~cm}^{-1}$ is assigned to carboxylic acid COO vibrations, originating either from unreacted starting material, or from the carboxylic acid on the monosodium terephthalate. Thermogravimetric analysis shows a main mass loss at ca. $300{ }^{\circ} \mathrm{C}$, in line with a monosodium salt, compared to thermally more stable disodium compounds. ${ }^{46}$ TGA also does
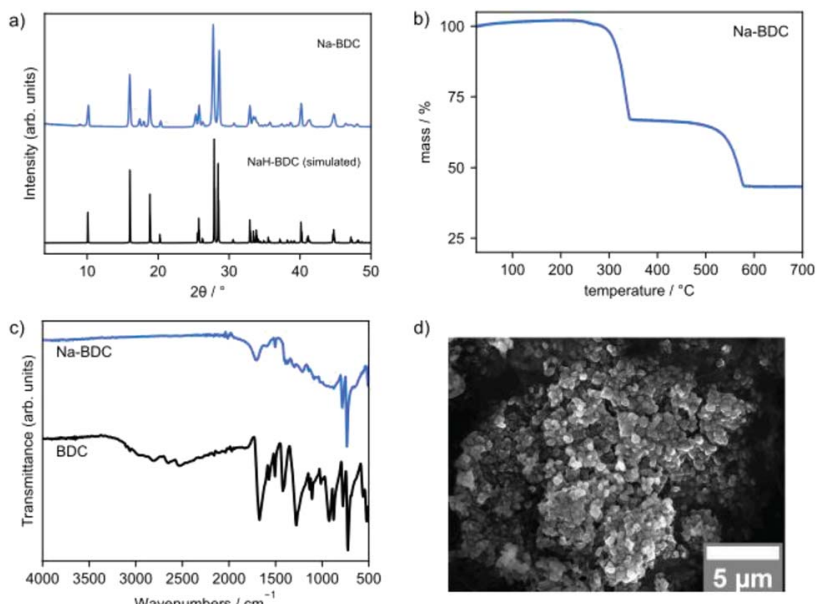

Fig. 3 Characterisation of mechanochemically prepared Na-BDC. (a) PXRD (simulated NaH-BDC from ref. 52), (b) TGA trace, (c) FTIR, and (d) SEM.

not indicate the presence of multiple materials, showing clear mass losses and stable plateaus (Fig. 3b) that are consistent with monosodium terephthalate. The identification of the material as NaH-BDC is further supported by elemental analysis (see Experimental section). The obtained material was also analyzed by SEM, which revealed expected agglomerates ( $>10$ $\mu \mathrm{m})$, consisting of primary particles with dimensions below 1 $\mu \mathrm{m}$ (Fig. 3d).

Although no liquid was added to the above-described synthesis, there is still water present in the form of crystal water of the hydrated sodium acetate used in the reaction. Demonstrating that the liquid is not required for the mechanochemical reaction itself, experiments were also conducted using anhydrous sodium acetate $(M / L=1)$. Assessing the reaction progression over time, PXRD patterns were collected at various time points (Fig. S2 $\dagger$ ). Interestingly, there is no discernible change throughout the studied time frame up to $4 \mathrm{~h}$ and product is already formed after only $15 \mathrm{~min}$ of milling. This reaction was also attempted using jars roughly double in size (internal volume of $25 \mathrm{~mL}$ instead of $14 \mathrm{~mL}$ ) as well as larger and heavier milling balls (12 mm diameter, $7 \mathrm{~g}$ ). Again, the product phase is formed after milling for $15 \mathrm{~min}$ and only small changes are observed upon increasing milling time to $90 \mathrm{~min}$.

Even though the target compound can be obtained without the use of any liquid at all, there are some health and safety concerns, especially at larger scales. The product of this neat milling procedure is a very fine powder, and it is strongly advised to use appropriate masks to prevent inhalation of the fine dust. By adding a small amount of water or using the hydrated sodium salt as a reagent these issues can be avoided. Other syntheses were carried out in the presence of a small amount of liquid originating from either source.

\section{Extension of the method to other sodium carboxylates}

Spurred on by the success of the reaction to prepare Na-BDC, several other organic carboxylic acids were treated similarly in 
a ball mill. Three different compounds were synthesised using 4,4'-biphenyldicarboxylic acid (BPDC), 4,4'-stilbenedicarboxylic acid (SDC), and 2,5-pyridinedicarboxylic acid (PDC), respectively. All of these have been previously reported as obtained from solution reaction and their potential as anode materials has been documented. ${ }^{\mathbf{4 8}-50}$

Attempts to synthesise Na-BPDC were again conducted in varying $\mathrm{M} / \mathrm{L}$ ratios and using water or ethanol as liquid additive (Fig. S3†). Comparison of PXRD patterns of obtained materials with previously published data by Choi et al. indicates the formation of both the monosodiated $\mathrm{NaH}-\mathrm{BPDC}$ as well as $\mathrm{Na}_{2}$ BPDC. ${ }^{48}$ Products of experiments with $\mathrm{M} / \mathrm{L}=2$ showed the presence of unreacted acid in FTIR spectra, thus the product of the experiment with $\mathrm{M} / \mathrm{L}=3$ and using ethanol as liquid additive was selected for further analysis and testing. Residual amounts of sodium acetate were initially considered negligible, however, both elemental analysis and electrochemical test runs revealed a substantial amount of electrochemically inactive components, most likely consisting of residual sodium acetate starting material. Thus, a simple washing step with ethanol was conducted to improve the sample purity. For this washed NaBPDC material, TGA shows only a small mass loss prior to $500{ }^{\circ} \mathrm{C}$, suggesting a high degree of conversion towards the disodium salt instead of a product of incomplete deprotonation. SEM analysis once more shows agglomerates, with primary particles of ill-defined inhomogeneous shape.

Using SDC as the carboxylic acid, the obtained sodium salt with $\mathrm{M} / \mathrm{L}=2$ exhibits a PXRD pattern roughly agreeing with previous reports, and showing some residual starting materials (Fig. S4 $\dagger)^{49}$ The two observed but not fully resolved low angle peaks potentially suggest the formation of both monosodiated and disodiated carboxylate species. Despite several adaptions to synthesis conditions ( $\mathrm{M} / \mathrm{L}$ ratio, liquid additive), no suitable product was obtained. Brief electrochemical test runs showed lower performance than expected, indicating that the product contained a considerable amount of electrochemically inactive material as was the case for Na-BPDC. A washing step with ethanol leads to a product showing a cleaner PXRD pattern and no sign of remaining sodium acetate. FTIR analysis of this material indicates the successful formation of Na-SDC with no sign of free acid groups. The TGA trace shows mass losses in the range of $250-400{ }^{\circ} \mathrm{C}$, suggesting the presence of a monosodium carboxylate species in addition to $\mathrm{Na}_{2}$-SDC. Elemental analysis also suggests some, albeit a small amount of impurity, however, the result overall is in line with $\mathrm{Na}_{2}$-SDC. Based on the results for Na-BDC and Na-BPDC, as well as following previously published data, ${ }^{\mathbf{4 9}}$ however, this sample was still deemed appropriate for electrochemical cycling studies as shown below. As with the previously described Na-carboxylates, the morphology of the material is that of large agglomerates where in the case of Na-SDC, the primary particles are rod-like with a length of up to $1 \mu \mathrm{m}$.

The targeted Na-PDC was also obtained using the above mechanochemical approach and analysed similarly (Fig. S5 $\dagger$ ). From the set of conducted experiments, optimised conditions were found to be $\mathrm{M} / \mathrm{L}=2$, ethanol as liquid additive, and $60 \mathrm{~min}$ of milling time as judged by PXRD pattern analysis of the products. Unreacted carboxylic acid is not observed in the obtained FTIR spectra due to the absence of $\mathrm{O}-\mathrm{H}$ bands around $3000 \mathrm{~cm}^{-1}$, confirming conversion of available organic reagent. A weak band at $1724 \mathrm{~cm}^{-1}$ may originate from a small amount of unreacted acid but bound to the bulk material. TGA analysis shows the main mass loss at $500{ }^{\circ} \mathrm{C}$, supportive of complete deprotonation of both carboxylic acids and the sole presence of a disodium salt. Similarly, the measured and calculated atom percentages agree well with a disodium pyridinedicarboxylate. In contrast to the three previously used acids, the nitrogen containing PDC appears to be more reactive, and the acidic protons more easily abstracted. This is thought to be caused by the nitrogen atom increasing the basicity of the system, thus aiding in the deprotonation reaction. ${ }^{53-55}$ SEM investigation reveals the material consists of irregularly shaped particles smaller than 1 micron, clumping together to larger agglomerates as seen for the other carboxylates.

Four different sodium carboxylate compounds are obtained using a rapid mechanochemical synthesis route. In addition to the time reduction, the important factor here is that all target materials can be obtained without bulk solvent and only reagent levels of liquid are used. This is considered to be a major improvement over conventional synthesis methods, with liquid waste reduced when using this mechanochemical approach, beneficial both in terms of cost but more importantly with respect to the principles of green chemistry. ${ }^{56,57}$ These conditions promise excellent applicability for large scale processing using, for example, extrusion techniques, in which both mechanosynthesis as well as a subsequent drying/heating step can occur concomitantly in a single processing unit. ${ }^{41,58-60}$

\section{Electrochemical testing of mechanochemically prepared organics}

Mechanochemically prepared sodium carboxylates were assessed as active materials in negative electrodes for sodium ion batteries. The experimental conditions for the specific samples tested can be found in the experimental section. Electrodes were prepared by mixing the active material, conductive carbon (Super C65) and an aqueous binder carboxymethylcellulose (CMC). As these organic solids are partially or fully soluble in water, PXRD patterns for the electrodes of all four compounds prior to cycling experiments were collected. All four of them exhibited retention of the parent phase confirming the active phase in the prepared electrode composites as the synthesised structure (Fig. 4a). SEM images for the electrodes before cycling revealed a homogeneous distribution of the active material crystallites with the carbon additive (Fig. 4b-e).

The electrochemical performance of the compounds was evaluated by assembling coin cells with sodium metal as the counter electrode. Cyclic voltammetry (CV) was initially recorded for 5 cycles between $0.01-2.5 \mathrm{~V}$ at a scan rate of $0.1 \mathrm{mV} \mathrm{s}^{-1}$. A single redox couple was observed for Na-BDC with a reduction peak at $\sim 0.14 \mathrm{~V}$ that shifted to the stable potential of $0.29 \mathrm{~V}$ in subsequent cycles (Fig. S6 $\dagger$ ). The oxidative peak at $0.55 \mathrm{~V}$ essentially remained unaltered during the experiment. A low 

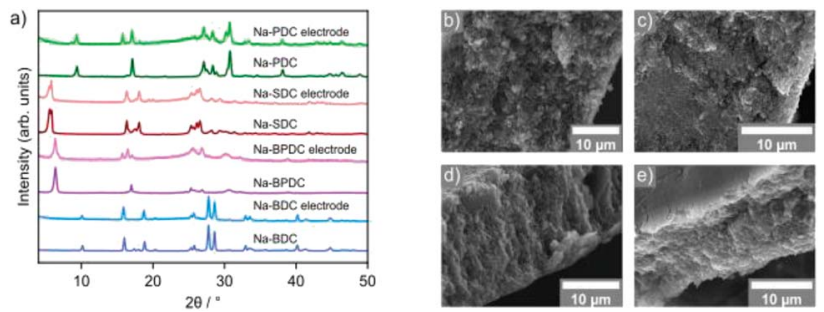

Fig. 4 Characterisation of electrode composites prior to electrochemical cycling experiments. PXRD patterns (normalised) of mechanochemically synthesised sodium carboxylates and the respective electrodes (a) and SEM images of electrodes made with (b) $\mathrm{Na}-\mathrm{BDC}$, (c) Na-BPDC, (d) Na-SDC, and (e) Na-PDC.

voltage minor redox couple was seen which could be ascribed to contribution from conductive carbon used in the preparation of the electrode (Fig. S7b†). Galvanostatic charge/discharge cycling was initially recorded at a current density of $100 \mathrm{~mA} \mathrm{~g}^{-1}$. The first discharge for Na-BDC exhibited a large irreversible capacity, corresponding to the solid-electrolyte interphase (SEI) formation, as seen commonly for anode materials. ${ }^{61,62}$ Additionally, the transformation of the monosodium terephthalate to the disodium form may contribute to this effect. ${ }^{46}$ For the subsequent cycle, a discharge capacity of $\sim 270 \mathrm{~mA} \mathrm{~h} \mathrm{~g}^{-1}$ was observed with a coulombic efficiency greater than $98 \%$ (Fig. 5a). The reversible capacity retention from the $2^{\text {nd }}$ cycle was seen to be $86 \%$ over 100 cycles. These values are in close agreement with the performance seen previously for Na-BDC synthesised by solution-based methods (Table S2 $\dagger$ ) ${ }^{44-46}$ The contribution to the capacity by the conductive carbon used in the preparation of electrode was estimated to be $\sim 37 \mathrm{~mA} \mathrm{~h} \mathrm{~g}^{-1}$ (Fig. S7 $\dagger$ ). Encouraged by these results, the rate performance was evaluated for this compound by increasing current rate from $25 \mathrm{~mA} \mathrm{~g}^{-1}$ to $1000 \mathrm{~mA} \mathrm{~g}^{-1}$ in 5 steps and back to $25 \mathrm{~mA} \mathrm{~g}^{-1}$. The compound fully recovered its capacity even after cycling at a high current density (Fig. 5b).

Pristine solid of Na-BPDC from mechanochemical reaction exhibited slightly lower cycling capacities (Fig. S8 †). Therefore, a washing step was introduced, and electrodes and other electrochemical studies were performed using the washed product. $\mathrm{CV}$ profiles exhibited a strong reduction peak at $0.2 \mathrm{~V}$ in the first discharge that shifted to a more positive potential and stabilised at $0.32 \mathrm{~V}$ in the subsequent cycles (Fig. S8 ${ }^{\dagger}$ ). The voltammogram comprised two oxidation peaks at $0.5 \mathrm{~V}$ and $0.65 \mathrm{~V}$ that
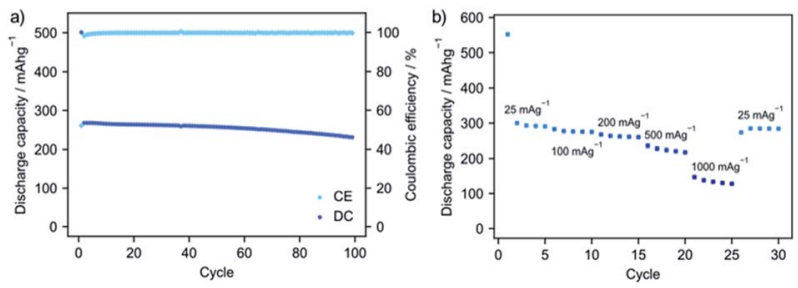

Fig. 5 (a) Galvanostatic cycling performance for Na-BDC at $100 \mathrm{~mA} \mathrm{~g}^{-1}$ cycled between 0.01-2.5 V. (b) Rate performance for $\mathrm{Na}$ $\mathrm{BDC}$, cycled 5 times at each current density. remained unaltered. These peaks suggested the presence of both the monosodiated and disodiated forms as seen in the earlier example. ${ }^{48}$ Galvanostatic cycling studies showed a first discharge capacity of $304 \mathrm{~mA} \mathrm{~h} \mathrm{~g}^{-1}$, that stabilised from the $2^{\text {nd }}$ cycle onwards with a capacity retention thereafter of $84 \%$ over 100 cycles (Fig. S8 $\dagger$ ). The coulombic efficiency required a few cycles before stabilising, which could be ascribed to the sodiation of the monosodium component. The material exhibited stable rate performance with complete recovery of capacities at $25 \mathrm{~mA} \mathrm{~g}^{-1}$ after step wise cycling up to a high current rate of $1 \mathrm{~A} \mathrm{~g}^{-1}$ (Fig. S9†).

Similar to Na-BPDC, for pristine Na-SDC we observed marginally lower specific capacity and its retention (Fig. S10†), indicating presence of an inactive component hindering cycling stability. Electrodes were consequently prepared after a washing step for the pristine material. The voltammogram, shown in Fig. S10, $\dagger$ exhibited a single step redox couple corresponding to the active material. A reduction peak at $0.33 \mathrm{~V}$ had a slight shift and stabilised at $0.39 \mathrm{~V}$ in the subsequent cycles. The oxidative peak seen at $0.67 \mathrm{~V}$ in the first cycle, remained relatively unchanged. It is, however, noteworthy that no noticeable humps or secondary peaks were observed from the second cycle onwards that were seen in the previous report. ${ }^{49}$ This implies absence of any by-product or amorphous solids and reiterates the advantage of mechanochemical synthesis in terms of cleaner conversion. During galvanostatic cycling a discharge capacity of $\sim 233 \mathrm{~mA} \mathrm{~h} \mathrm{~g}^{-1}$ for the $2^{\text {nd }}$ cycle was observed, with a moderately stable capacity retention over 100 cycles (Fig. S10†) ${ }^{49}$ The rate performance for the compound exhibited complete retention of the capacity at low rate $\left(25 \mathrm{~mA} \mathrm{~g}^{-1}\right)$, after cycling over multiple current densities (Fig. S11†). Notably, the cycling stability and capacities for Na-SDC are comparable to the corresponding previous study, despite reduction in the amount of carbon additive (Table S2 $\dagger$ ).

Unlike other examples in this work, the CV profile for NaPDC had two discernible peaks in both the reduction and oxidation cycles (Fig. S12†). As reported earlier by Padhy et al., this corresponds to insertion of $\mathrm{Na}^{+}$ions in two steps involving. the interaction with the pyridyl $\mathrm{N}$-atom at higher voltage and subsequent insertion to the carboxylate group at lower potential. ${ }^{50}$ During galvanostatic cycling a relatively low irreversible capacity and much higher coulombic efficiency $(75 \%)$ was seen for the first cycle (Fig. S12 $\dagger$ ). In the following cycle, a discharge capacity of $\sim 258 \mathrm{~mA} \mathrm{~h} \mathrm{~g}{ }^{-1}$ was observed which was highly reversible and reasonably stable over 100 cycles. This compound also demonstrated very stable rate performance with almost complete recovery of capacity at $25 \mathrm{~mA} \mathrm{~g}^{-1}$ after cycling in multiple steps up to $1 \mathrm{~A} \mathrm{~g}^{-1}$ (Fig. S13†). It is also worth noting that in the current work the capacity retention is achieved with the use of CMC as the binder and lower content of conductive carbon (Table S2 $\dagger$ ). Avoiding the previously discussed issues accompanying the use of PVDF which has been used as binder in published reports while displaying a comparable electrochemical performance is to be considered a promising aspect in attempting to make the production of these anode materials less hazardous. 
Mechanochemistry and organic anodes - a green solution for sodium ion batteries

One of the main goals of current research on rechargeable battery technology is to provide more sustainable and environmentally friendly components and methods for energy storage devices. Valorization of biomass has been shown to be a great source of building block chemicals, rendering the environmental impact of products much lower. ${ }^{63-65}$ The missing part of the puzzle is to develop energy efficient processes to convert these organic compounds into their active forms, usable for a given application. The methodology developed in this work represents a great step forward in this respect, as it is not only able to produce the desired sodium carboxylate phases in very short reaction times but does so in a very energy-efficient manner with the additional benefit of not requiring solvent. The latter point is of great importance with respect to production of waste, again contributing to an overall greener process. The reduction of preparation time in combination with scaling capabilities inherent to mechanochemical processing satisfies demands for large-scale production. Since the production of electrode materials makes up around half of the total cost of a battery, ${ }^{4}$ finding cheaper and faster ways of synthesizing and preparing the active phase contributes to lowering the total cost of device. Moving forward to the preparation of functioning electrode composites, the environmentally benign binder Na-CMC has been used in this work. Besides being obtainable from biomass like the carboxylic acids, it requires only water as solvent, keeping in line with the goal of providing sustainable solutions for each component of the electrode. ${ }^{23}$

The mechanochemical methodology applied in this project should serve as a blueprint for further studies. The variety of used carboxylic acids, including nitrogen containing PDC, demonstrates the versatility of the method and it promises to be applicable for other reagents.

\section{Conclusions}

In this study, we have demonstrated efficient mechanochemical synthesis of sodium dicarboxylate materials and showcased their applicability as anode materials for sodium ion batteries. Four sodium carboxylates with varying chemical characteristics were mechanochemically prepared and evaluated for sodium-ion storage. All four compounds exhibited equivalent electrochemical performance to the previously reported materials synthesised using conventional methods. The fast and environmentally friendly synthesis method is thought to be easily translatable to large scale production of these compounds. It is also a very important step towards completing the so-called green battery cycle, with complete recyclability of involved chemicals as well as no waste accumulation during production.

Electrochemistry and performance of organic electrode materials is still at an early stage of practical applications and somewhat less competitive to the benchmark hard carbon, but apart from inherent advantages of being environmentally friendly, such widely applicable and facile preparation methods of active materials can only spur interest and facilitate its development.

\section{Author contributions}

A. R. A. and R. E. M. designed the project. D. N. R. synthesised and characterised materials. A. V. D. performed electrode preparation, and electrochemical testing. D. N. R. and A. V. D. wrote the manuscript. All authors edited and reviewed the manuscript.

\section{Conflicts of interest}

There are no conflicts to declare.

\section{Acknowledgements}

D. N. R. acknowledges funding through the EPSRC (EP/ N509759/1). The authors thank the Faraday Institution for funding (Grant FIRG018). The authors also acknowledge the EPSRC Light Element Facility Grant (EP/T019298/1) and the EPSRC Strategic Equipment Resource Grant (EP/R023751/1).

\section{Notes and references}

1 IEA, Key World Energy Stat., 2020, https:/www.iea.org/ reports/key-world-energy-statistics-2020.

2 M. Li, J. Lu, Z. Chen and K. Amine, Adv. Mater., 2018, 30, 1800561.

3 M. D. Slater, D. Kim, E. Lee and C. S. Johnson, Adv. Funct. Mater., 2013, 23, 947-958.

4 C. Vaalma, D. Buchholz, M. Weil and S. Passerini, Nat. Rev. Mater., 2018, 3, 18013.

5 J. Xiang, L. Yang, L. Yuan, K. Yuan, Y. Zhang, Y. Huang, J. Lin, F. Pan and Y. Huang, Joule, 2019, 3, 2334-2363.

6 N. Tapia-Ruiz, A. R. Armstrong, H. Alptekin, M. A. Amores, H. Au, J. Barker, R. Boston, W. R. Brant, J. M. Brittain, Y. Chen, M. Chhowalla, Y.-S. Choi, S. I. R. Costa, M. Crespo Ribadeneyra, S. A. Cussen, E. J. Cussen, W. I. F. David, A. V. Desai, S. A. M. Dickson, E. I. Eweka, J. D. Forero-Saboya, C. P. Grey, J. M. Griffin, P. Gross, X. Hua, J. T. S. Irvine, P. Johansson, M. O. Jones, M. Karlsmo, E. Kendrick, E. Kim, O. V Kolosov, Z. Li, S. F. L. Mertens, R. Mogensen, L. Monconduit, R. E. Morris, A. J. Naylor, S. Nikman, C. A. O'Keefe, D. M. C. Ould, R. G. Palgrave, P. Poizot, A. Ponrouch, S. Renault, E. M. Reynolds, A. Rudola, R. Sayers, D. O. Scanlon, S. Sen, V. R. Seymour, B. Silván, M. T. Sougrati, L. Stievano, G. S. Stone, C. I. Thomas, M.-M. Titirici, J. Tong, T. J. Wood, D. S. Wright and R. Younesi, J. Phys.: Energy, 2021, 3, 031503.

7 H. Kim, H. Kim, Z. Ding, M. H. Lee, K. Lim, G. Yoon and K. Kang, Adv. Energy Mater., 2016, 6, 1600943.

8 Y. Liu, X. Liu, T. Wang, L.-Z. Fan and L. Jiao, Sustainable Energy Fuels, 2017, 1, 986-1006.

9 A. Manthiram, Nat. Commun., 2020, 11, 1550. 
10 M. Li, J. Lu, X. Ji, Y. Li, Y. Shao, Z. Chen, C. Zhong and K. Amine, Nat. Rev. Mater., 2020, 5, 276-294.

11 Y. Li, Y. Lu, P. Adelhelm, M.-M. Titirici and Y.-S. Hu, Chem. Soc. Rev., 2019, 48, 4655-4687.

12 T. Perveen, M. Siddiq, N. Shahzad, R. Ihsan, A. Ahmad and M. I. Shahzad, Renewable Sustainable Energy Rev., 2020, 119, 109549.

13 K. Holguin, M. Mohammadiroudbari, K. Qin and C. Luo, J. Mater. Chem. A, 2021, 9, 19083-19115.

14 B. Xiao, T. Rojo and X. Li, ChemSusChem, 2019, 12, 133-144.

15 D. Chen, W. Zhang, K. Luo, Y. Song, Y. Zhong, Y. Liu, G. Wang, B. Zhong, Z. Wu and X. Guo, Energy Environ. Sci., 2021, 14, 2244-2262.

16 A. V. Desai, R. E. Morris and A. R. Armstrong, ChemSusChem, 2020, 13, 4866-4884.

17 J. J. Shea and C. Luo, ACS Appl. Mater. Interfaces, 2020, 12, 5361-5380.

18 P. Poizot, J. Gaubicher, S. Renault, L. Dubois, Y. Liang and Y. Yao, Chem. Rev., 2020, 120, 6490-6557.

19 B. Esser, F. Dolhem, M. Becuwe, P. Poizot, A. Vlad and D. Brandell, J. Power Sources, 2021, 482, 228814.

20 Y. Bai, C. Liu, Y. Shan, T. Chen, Y. Zhao, C. Yu and H. Pang, Adv. Energy Mater., 2021, 2100346.

21 Y. Wei, L. Zou, H. Wang, Y. Wang and Q. Xu, Adv. Energy Mater., 2021, 2003970.

22 T. P. Nguyen, A. D. Easley, N. Kang, S. Khan, S.-M. Lim, Y. H. Rezenom, S. Wang, D. K. Tran, J. Fan, R. A. Letteri, X. He, L. Su, C.-H. Yu, J. L. Lutkenhaus and K. L. Wooley, Nature, 2021, 593, 61-66.

23 D. Bresser, D. Buchholz, A. Moretti, A. Varzi and S. Passerini, Energy Environ. Sci., 2018, 11, 3096-3127.

24 J.-L. Do and T. Friščić, ACS Cent. Sci., 2017, 3, 13-19.

25 T. Friščić, C. Mottillo and H. M. Titi, Angew. Chem., Int. Ed., 2020, 59, 1018-1029.

26 S. Mateti, M. Mathesh, Z. Liu, T. Tao, T. Ramireddy, A. M. Glushenkov, W. Yang and Y. I. Chen, Chem. Commun., 2021, 57, 1080-1092.

27 D. Tan and T. Friščić, Eur. J. Org. Chem., 2018, 2018, 18-33. 28 J. Andersen and J. Mack, Green Chem., 2018, 20, 1435-1443. 29 D. Tan and F. García, Chem. Soc. Rev., 2019, 48, 2274-2292.

30 V. Šepelák, A. Düvel, M. Wilkening, K.-D. Becker and P. Heitjans, Chem. Soc. Rev., 2013, 42, 7507.

31 G. Majano, L. Borchardt, S. Mitchell, V. Valtchev and J. PérezRamírez, Microporous Mesoporous Mater., 2014, 194, 106114.

32 B. Szczęśniak, S. Borysiuk, J. Choma and M. Jaroniec, Mater. Horiz., 2020, 7, 1457-1473.

33 D. N. Rainer and R. E. Morris, Dalton Trans., 2021, 50, 89959009.

34 D. Tan, L. Loots and T. Friščić, Chem. Commun., 2016, 52, 7760-7781.

35 M. J. Muñoz-Batista, D. Rodriguez-Padron, A. R. PuenteSantiago and R. Luque, ACS Sustainable Chem. Eng., 2018, 6, 9530-9544.

36 K. Wang, Y. Wang, J. Wang, Y. Gan, X. He, Y. Xia, J. Zhang, H. Huang, W. Zhang and C. Liang, Energy Fuels, 2021, 35, 4596-4603.
37 R. Schlem, C. F. Burmeister, P. Michalowski, S. Ohno, G. F. Dewald, A. Kwade and W. G. Zeier, Adv. Energy Mater., 2021, 11, 2101022.

38 T. Friščić, in Encyclopedia of Inorganic and Bioinorganic Chemistry, John Wiley \& Sons, Ltd, Chichester, UK, 2014, pp. 1-19.

39 T. Stolar and K. Užarević, CrystEngComm, 2020, 22, 45114525.

40 E. R. Engel and J. L. Scott, Green Chem., 2020, 22, 3693-3715. 41 M. Rubio-Martinez, C. Avci-Camur, A. W. Thornton, I. Imaz, D. Maspoch and M. R. Hill, Chem. Soc. Rev., 2017, 46, 34533480 .

42 C. Li, L. Liu, J. Kang, Y. Xiao, Y. Feng, F.-F. Cao and H. Zhang, Energy Storage Mater., 2020, 31, 115-134.

43 L. Wang, Y. Han, X. Feng, J. Zhou, P. Qi and B. Wang, Coord. Chem. Rev., 2016, 307, 361-381.

44 Y. Park, D.-S. S. Shin, S. H. Woo, N. S. Choi, K. H. Shin, S. M. Oh, K. T. Lee and S. Y. Hong, Adv. Mater., 2012, 24, 3562-3567.

45 L. Zhao, J. Zhao, Y.-S. S. Hu, H. Li, Z. Zhou, M. Armand and L. Chen, Adv. Energy Mater., 2012, 2, 962-965.

46 A. Abouimrane, W. Weng, H. Eltayeb, Y. Cui, J. Niklas, O. Poluektov and K. Amine, Energy Environ. Sci., 2012, 5, 9632.

47 M. Armand, S. Grugeon, H. Vezin, S. Laruelle, P. Ribière, P. Poizot and J. M. Tarascon, Nat. Mater., 2009, 8, 120-125.

48 A. Choi, Y. K. Kim, T. K. Kim, M.-S. Kwon, K. T. Lee and H. R. Moon, J. Mater. Chem. A, 2014, 2, 14986-14993.

49 C. Wang, Y. Xu, Y. Fang, M. Zhou, L. Liang, S. Singh, H. Zhao, A. Schober and Y. Lei, J. Am. Chem. Soc., 2015, 137, 3124-3130.

50 H. Padhy, Y. Chen, J. Lüder, S. R. Gajella, S. Manzhos and P. Balaya, Adv. Energy Mater., 2018, 8, 1701572.

51 I. Halasz, S. A. J. J. Kimber, P. J. Beldon, A. M. Belenguer, F. Adams, V. Honkimäki, R. C. Nightingale, R. E. Dinnebier and T. Friščić, Nat. Protoc., 2013, 8, 17181729.

52 S. H. Dale and M. R. J. Elsegood, Acta Crystallogr., Sect. C: Cryst. Struct. Commun., 2003, 59, m475-m477.

53 G. E. Dunn, G. K. J. Lee and H. Thimm, Can. J. Chem., 1972, 50, 3017-3027.

54 E. Norkus, E. Gaidamauskas, I. Stalnionienė and D. C. Crans, Heteroat. Chem., 2005, 16, 285-291.

55 Z. W. Luo and S. Y. Lee, Nat. Commun., 2017, 8, 15689.

56 P. T. Anastas and J. C. Warner, Green Chemistry: Theory and Practice, Oxford University Press, 2000.

57 K. J. Ardila-Fierro and J. G. Hernández, ChemSusChem, 2021, 14, 2145-2162.

58 D. E. Crawford and J. Casaban, Adv. Mater., 2016, 28, 57475754.

59 D. E. Crawford, C. K. G. Miskimmin, A. B. Albadarin, G. Walker and S. L. James, Green Chem., 2017, 19, 1507-1518.

60 J. Casaban, Y. Zhang, R. Pacheco, C. Coney, C. Holmes, E. Sutherland, C. Hamill, J. Breen, S. L. James, D. Tufano, D. Wong, E. Stavrakakis, H. Annath and A. Moore, Faraday Discuss., 2021, 231, 312-325. 
61 E. Peled and S. Menkin, J. Electrochem. Soc., 2017, 164, 64 L. Wu, T. Moteki, A. A. Gokhale, D. W. Flaherty and A1703-A1719.

62 C. Bommier and X. Ji, Small, 2018, 14, 1703576.

63 R. A. Sheldon, Green Chem., 2014, 16, 950-963.

F. D. Toste, Chem, 2016, 1, 32-58.

65 M. Volanti, D. Cespi, F. Passarini, E. Neri, F. Cavani, P. Mizsey and D. Fozer, Green Chem., 2019, 21, 885-896. 